\title{
LA VARIABILIDAD ESPACIAL DE LAS TEMPERATURAS EN LA PENÍNSULA IBÉRICA EXPLICADA POR LOS TIPOS DE TIEMPO DE CIRCULACIÓN ZONAL: ESTE Y OESTE. UN POSIBLE EFECTO FOEHN A ESCALA PENINSULAR
}

\author{
Dhais PEÑA ANGULO ${ }^{1-2}$, José Carlos GONZÁLEZ HIDALGO ${ }^{1-2}$, \\ Ricardo TRIGO ${ }^{3}$, Nicola CORTESI ${ }^{4}$ \\ ${ }^{1}$ Departamento de Geografía, Universidad de Zaragoza, España. \\ ${ }^{2}$ Instituto de Investigación en Ciencias Ambientales de Aragón (IUCA), Universidad de Zaragoza, \\ España. \\ ${ }^{3}$ Instituto de Geofisica "Infante D. Luiz", Universidad de Lisboa, Portugal. \\ ${ }^{4}$ Centro Nacional de Supercomputación, Barcelona, España. \\ dhaispa@gmail.com, jcgh@unizar.es, rmtrigo@fc.ul.pt, nicolacortesi@ymail.com
}

\section{RESUMEN}

Los tipos de tiempo se definen como situaciones sinópticas específicas, clasificadas según diversos criterios. La clasificación de Jeckinson y Collison es automática, y emplea datos de presión de superficie y diversos algoritmos que definen 26 tipos de tiempo. Se ha estudiado la relación entre los tipos de tiempo y las temperaturas mensuales de la Península Ibérica de la base de datos "MOnthly TEmperature DAtabase of Spain". El análisis de la relación muestra una respuesta asimétrica entre las costas este y oeste peninsular en presencia de advecciones atlánticas o mediterráneas. Así, las advecciones del Oeste favorecen el descenso de temperaturas en la fachada atlántica del territorio, mientras que en la costa mediterránea se observa un aumento de las mismas. Sin embargo, cuando tiene lugar las advecciones del Este ocurre lo contrario, descenso de temperatura en la costa mediterránea y aumento térmico en la fachada atlántica. La distribución espacial de las principales cadenas montañosas parece ser la clave del reparto espacial de las temperaturas y del efecto foehn que tiene lugar a gran escala en la Península Ibérica.

Palabras clave: Temperaturas, Variabilidad, Efecto Foehn, España.

\begin{abstract}
Weather types are defined as specific synoptic situations that can be classified using several criteria. Jeckinson and Collison's classification is an automatic algorithm that uses surface pressure data to define 26 weather types. In this study, the relationship between weather types and monthly temperatures has been studied for the Iberian Peninsula, based on data from the "MOnthly TEmperature DAtabase of Spain" database. This analysis shows an asymmetric behaviour in the East and West coasts of the peninsula in response to Mediterranean and Atlantic advection. A west advection leads to decreases in temperatures in the Atlantic facade of the studied territory, while an increase of temperatures is observed along the Mediterranean coast. With an East advection the opposite phenomenon occurs: temperatures decay on the Mediterranean coast and increase in the Atlantic facade. The spatial distribution of the
\end{abstract}


main mountain chains in the Iberian Peninsula appears to be the key element explaining the spatial distribution of temperatures and the observed Foehn's effect occurring at a large scale in this territory.

Key words: Temperatures, Variability, Foehn effect, Spain.

\section{INTRODUCCIÓN}

En un ascenso, toda masa de aire reduce su temperatura adiabáticamente por efecto de la disminución de presión. Este fenómeno físico tiene amplio reconocimiento en climatología e identifica vientos característicos generadores de sombras pluviométricas a sotavento de cadenas montañosas transversales a las direcciones dominantes de los flujos, que reciben nombres bien conocidos: Fohen (Alemania y Austria), Chinook (Canadá y EEUU), Zonda (Argentina), Santa Ana (Suroeste EEUU), Canterbury-nor'wester' (Nueva Zelanda), "berg wind" (Sudáfrica), "fagüeño" (Aragón) etc. Entre sus efectos se citan situaciones relacionadas con olas de calor (Gaffin, 2002; Takane et al., 2015), polución atmosférica (Natale et al., 1999; Li et al., 2015), riesgo y propagación de incendios (Sharples et al., 2010; Jin et al., 2014), deshielo (Soderberg y Parmhed, 2006; Pedersen et al., 2015), entre otros. El calentamiento adiabático por descenso de la masa de aire describe en términos físicos el fenómeno, pero su distribución espacial, intensidad y frecuencia dependen de las características topográficas y de las condiciones de circulación atmosférica (Seluchi et al., 2003).

El estudio de la relación entre los tipos de tiempo y las temperaturas durante la segunda mitad del siglo 20 en la Península Ibérica ha permitido catalogar las direcciones dominantes desde 1950 hasta el presente y analizar su relación con las temperaturas en una escala mensual (Peña-Angulo et al., 2016). Los resultados más señalados de dicha investigación muestran la existencia de un asimetría en el comportamiento, fundamentalmente, de las temperaturas máximas entre las fachadas mediterráneas y atlánticas, es decir una relación negativa/positiva en las fachadas oeste/este en condiciones de flujo del atlántico (oeste), y viceversa en condiciones de flujo del mediterráneo (este). Este hecho sugiere que las temperaturas de las dos fachadas marítimas de la Península Ibérica, expuestas a flujos zonales procedentes del Atlántico o del Mediterráneo, pudieran reflejar en una escala regional el proceso adiabático, cuyo origen estaría en la disposición transversal del Sistema Ibérico y Bético casi paralelo a la fachada mediterránea. Por otra parte, esta hipótesis sugeriría además que en las zonas interiores hasta las vertientes occidentales del Sistema Ibérico el efecto de los flujos zonales seguiría el patrón de la costa atlántica con las lógicas variaciones impuestas preferentemente por la altitud.

El clima de la Península Ibérica está condicionado por su posición en la zona de transición subtropical de la fachada occidental europea. Esta localización además se ve fuertemente afectada por su posición entre dos masas de agua muy contrastadas, el Atlántico y Mediterráneo, y por una distribución de sus principales cadenas montañosas en bandas paralelas oeste-este que configuran tres grandes ámbitos climáticos: la costa norte, el centro-oeste que se prolonga hasta la costa sur, y la fachada mediterránea y su penetración por el noreste en el valle del Ebro. El gran espacio interior se subdivide en dos grandes unidades, denominadas Meseta Norte y Sur, y presenta una altitud notable, con valores por encima de los $600 \mathrm{~m}$ osl en la Meseta Norte y en 
torno a los $400 \mathrm{~m}$ osl en la Meseta sur en suave descenso hacia el oeste en territorio portugués. La distribución espacial de las principales cadenas montañosas parece ser la clave del reparto espacial de las temperaturas y del efecto foehn que tiene lugar a gran escala en la Península Ibérica. Los valores de temperatura se ven condicionados por la latitud, altitud, distancia a la costa y corrientes marinas, pero también por las características del relieve. La topografía local determina la variabilidad espacial de las temperaturas, así en áreas de extensa llanura los valores similares de temperatura se extienden más que en zonas accidentadas con barreras montañosas en las que se aprecia pendientes de solana y umbría, ladera de barlovento o sotavento que tiene su efecto en las temperaturas, vegetación hidrología, entre otros.

En el presente estudio se analiza espacialmente la relación entre los tipos de tiempo y las temperaturas mensuales. El objetivo del presente estudio es identificar si los promedios mensuales de temperaturas en condiciones de flujo zonal difieren entre fachadas, y si las áreas del interior se comportan del mismo modo que las costas atlánticas y mediterráneas.

\section{DATOS Y MÉTODOLOGÍA}

El presente estudio emplea en el territorio peninsular español la versión de malla de alta resolución a $10 \mathrm{~km}^{2}$ de la base de datos MOTEDAS (Gonzalez-Hidalgo et al. 2015). Dicha malla se ha creado tras realizar un control de calidad y reconstrucción de 1358 observatorios procedentes de los fondos documentales de la AEMet (Agencia Estatal de Meterología). Para el territorio portugués la información disponible es menor, y solamente hemos podido contar con 28 observatorios procedentes del Instituto Portugués de Mar y Atmosfera (IPMA) a los que se aplicó el mismo proceso de control de calidad y reconstrucción que en MOTEDAS (Fig. 1). La menor densidad de información en el territorio portugués nos ha sugerido no realizar una ampliación de la malla por lo que dicha información se utiliza en su localización original (coordenadas de los observatorios), y se ha empleado para contrastar la coherencia espacial entre ambos países, en una variable natural que no entiende de fronteras.

Los tipos de tiempo se analizaron utilizando la base de datos subdiaria de presiones en superficie procedente de NCAR/NCEP cuya resolución espacial es de $2^{\circ}$ (Kistler et al., 2001), y que tiene la ventaja de empezar en 1948 respecto a las más recientes Era-Interim, Era-40, y MERRA. En la Península Ibérica, debido a la compleja dinámica atmosférica comentada no existe una clasificación de las situaciones sinópticas comúnmente aceptada (Martín-Vide 2002), si bien los trabajos más recientes (Spellman 2000; Trigo \& DaCamara 2000; Martín-Vide 2002; Cortesi et al. 2013b; Santurtún et al. 2015) han aplicado la modificación de Trigo \& DaCamara (2000) de la clasificación de Jenkinson \& Collison (1977).

Brevemente, la clasificación se aplica a los datos de presión diaria en una malla de 16 pixel procedentes de la base de datos NCEP/NCAR Reanalysis (periodo 19512010) con centro en la PI. La clasificación calcula 6 índices geostróficos que tienen en cuenta la dirección (D), la fuerza $(\mathrm{F})$ y la vorticidad del viento $(\mathrm{Z})$, y posteriormente se aplican una serie de reglas que permiten obtener 26 tipos de tiempo, 8 direccionales: Norte (N), Sur (S), Este (E), Oeste (W), Noreste (NE), Noroeste (NW), Sureste (SE), y Suroeste (SW), 2 puros: Anticiclón (A) y Ciclónico (C), y la combinación 
de ambos tipos en los llamados híbridos: AN, AS, AE, AW, ANE, ANW, ASE, ASW, CN, CS, CE, CO, CNE, CNW, CSE, CSW. Las ecuaciones y reglas empleadas están definidas en Trigo \& DaCamara (2000) y aplicadas en diferentes trabajos (Cortesi et al., 2013a; Peña-Angulo, et al. 2016). La clase sin catalogar indicada por Martín Vide (2002) fue diseminada en las otras 26 al igual que Trigo \& DaCamara (2000).
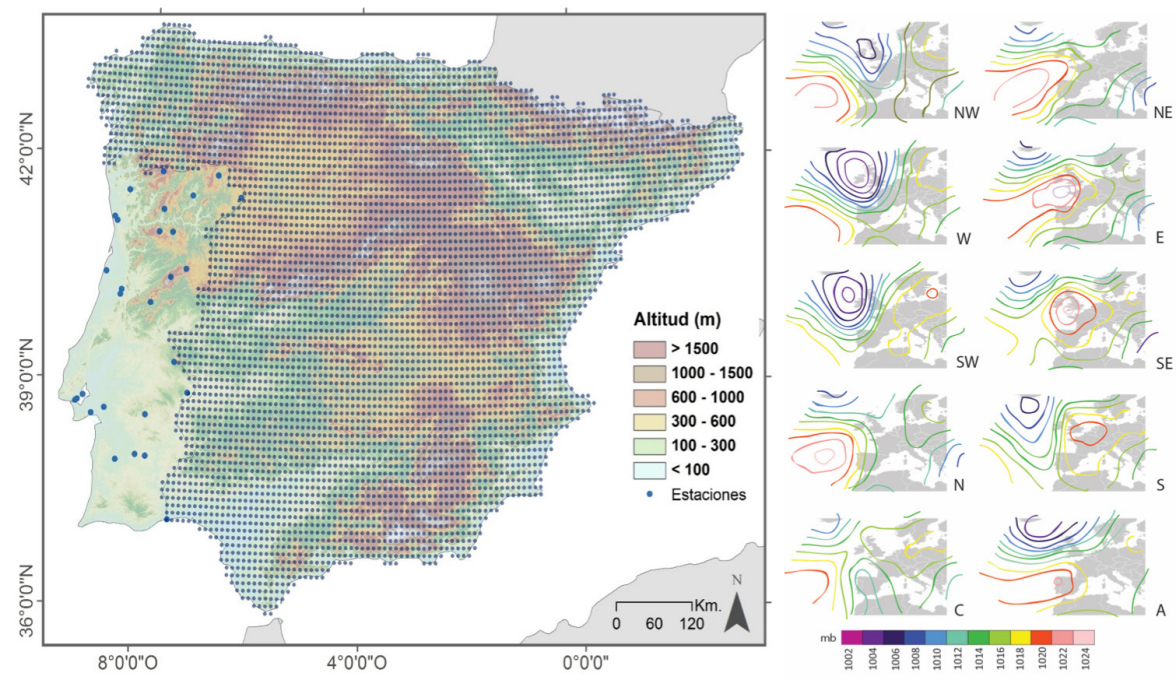

Fig 1: Distribución espacial de los datos de temperatura en la Península Ibérica (izquierda). Principales tipos de tiempo (derecha).

La relación entre los tipos de tiempo y las temperaturas se realizó mediante el coeficiente de Pearson (r) que expresa la fuerza y signo de la correlación entre variables. En el presente trabajo se estudió la distribución espacial de la correlación entre la frecuencia mensual de los tipos de tiempo Este y Oeste y la Tmax y Tmin mensual. Estos dos flujos Oeste y Este a diferencia de los otros tipos de tiempo mostraban una correlación tanto positiva como negativa en un mismo mes lo que promovió el interés de su análisis espacial, fundamentalmente en Tmax. Los otros tipos de tiempo presentan un alto porcentaje de contribución positiva si tienen un origen sur, y negativa si tienen un origen norte. Por otra parte los tipos de tiempo puro muestran sus particularidades, pero en general con el anticiclón tiene lugar un aumento de las temperaturas y con el ciclónico una disminución de las mismas.

Para corroborar los resultados obtenidos con los datos mensuales, se utilizó la información diaria de temperatura proceden de la base de datos ECA (European Climate Assessment \& Dataset), cuyos originales españoles a su vez proceden de los fondos documentales de AEMet. Los datos han superado un control de calidad y se refieren todos ellos a observatorios de primer orden localizados en capitales de provincia o país (Lisboa, Madrid y Valencia). En cada observatorio se calculo la media de las temperaturas máximas en los días con tipo de tiempo del Oeste y Este, así como el promedio mensual global. 


\section{RESULTADOS Y DISCUSIÓN}

Las advecciones del Este y Oeste presentan una mayor variabilidad espacial en sus efectos respecto a los otros tipos de tiempo, y el reparto de sus zonas de influencia sugiere entre otras causas el efecto del arco montañoso que separa la fachada mediterránea al este del resto peninsular. De este modo en un mismo mes se puede tener contribución positiva y negativa significativa, dependiente de la dirección del flujo, en áreas extensas.

El flujo del Este (Fig. 2) se correlaciona de manera significativa y negativa con Tmax y Tmin en la fachada mediterránea y presenta correlación positiva y significativa en la margen atlántica en los meses cálidos de abril a septiembre. Este comportamiento es más extendido y evidente en Tmax que en Tmin ya que también ocurre en marzo y octubre, cuando en Tmin. En los meses de invierno se observa una correlación negativa, que en el caso de la Tmax sólo es significativa al este del territorio y en el valle del Ebro; mientras que en Tmin la correlación es significativa y negativa en todo el territorio. En resumen, a excepción de los meses fríos, el flujo del Este muestra un gradiente de este a oeste, pasando de efectos negativos a positivos lo que sugiere un efecto refrescante en la costa por la entrada de masas de aire procedentes del mar Mediterráneo y un calentamiento adiabático traspasadas las cordilleras paralelas a la costa en el interior peninsular.


Fig 2: Significación (p-valor) de la correlación (r) entre Tmax y Tmin mensual y el tipo de tiempo Este en la Península Ibérica

Una situación distinta se observa con el flujo del Oeste (Fig. 3). Tanto Tmax como Tmin en los meses cálidos de abril a septiembre, presentan correlación negativa y significativa en la costa atlántica y el interior con advecciones atlánticas, mientras que la correlación es positiva y significativa en la costa mediterránea. Al igual que ocurre con la advección del Este esta relación afecta a un mayor espacio y es mucho más clara y evidente en Tmax que en Tmin. En el caso de la Tmax, durante los meses fríos de noviembre a marzo se observa el mismo patrón que en el resto de los meses, a excepción de diciembre que presenta una correlación positiva y significativa en gran 
parte del territorio. En estos meses fríos a diferencia del tipo de tiempo del Este, la Tmin muestra una correlación positiva y significativa en todo el territorio.
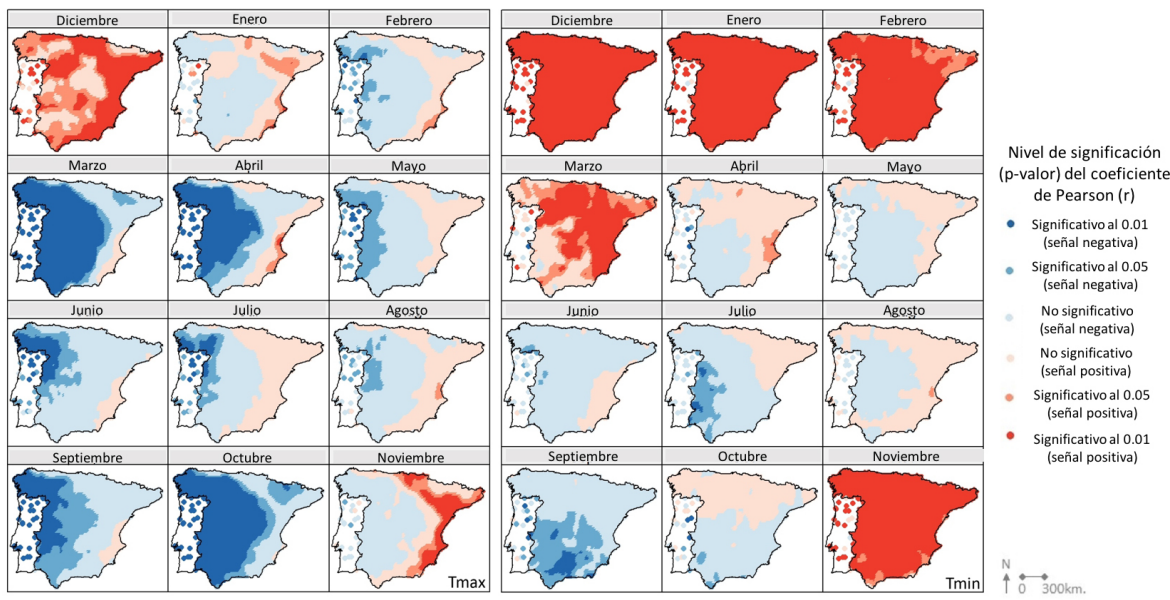

Fig 3: Significación (p-valor) de la correlación (r) entre la Tmax y Tmin mensual y el tipo de tiempo Oeste en la Península Ibérica

Los resultados mostrados parecen sugerir en consecuencia un efecto fohen generalizado en la Península Ibérica sugerido en muy pocas ocasiones (Fernández-Montes et al., 2012), si bien proceden de un análisis de promedios mensuales totales. En la Tabla 1 se muestran los promedios de mensuales de Tmax calculados a partir de los días clasificados como advecciones Oeste, Este y los promedios mensuales de los observatorios de control en la fachada oeste (Lisboa), en el centro del área de estudio (Madrid) y en la costa mediterránea (Valencia). Las tres ciudades se localizan en latitudes muy próximas y se diferencian por su posición latitudinal.

\begin{tabular}{|l|l|r|c|c|c|c|c|c|c|c|c|c|r|}
\hline \multicolumn{2}{|c|}{} & Ene & Feb & Mar & Abr & May & Jun & Jul & Ago & Sep & Oct & Nov & Dic \\
\hline \multirow{3}{*}{ Lisboa } & Este & 13,0 & 15,4 & 19,3 & 22,1 & 25,8 & 28,2 & 30,3 & 30,8 & 29,0 & 24,3 & 17,8 & 13,4 \\
\cline { 2 - 14 } & Oeste & 15,4 & 15,6 & 16,1 & 17,3 & 18,9 & 21,3 & 23,5 & 24,7 & 22,8 & 20,2 & 17,9 & 16,1 \\
\cline { 2 - 14 } & Media & 14,6 & 15,9 & 18,1 & 19,7 & 22,1 & 25,3 & 27,8 & 28,0 & 26,3 & 22,4 & 17,9 & 14,9 \\
\hline \multirow{3}{*}{ Madrid } & Este & 9,1 & 11,0 & 15,7 & 19,4 & 24,1 & 29,0 & 32,0 & 31,1 & 27,1 & 20,0 & 13,3 & 9,4 \\
\cline { 2 - 13 } & Oeste & 9,4 & 10,5 & 13,0 & 15,5 & 17,8 & 21,8 & 28,1 & 27,6 & 22,1 & 16,6 & 12,3 & 10,2 \\
\cline { 2 - 13 } & Media & 9,4 & 11,5 & 15,3 & 17,8 & 21,9 & 27,0 & 31,2 & 30,4 & 25,8 & 18,9 & 13,0 & 9,7 \\
\hline \multirow{3}{*}{ Valencia } & Este & 13,0 & 14,1 & 17,0 & 19,8 & 23,0 & 27,1 & 29,2 & 29,3 & 26,8 & 22,1 & 17,1 & 13,9 \\
\cline { 2 - 12 } & Oeste & 17,0 & 18,3 & 20,7 & 22,8 & 25,7 & 29,4 & 31,5 & 32,4 & 29,2 & 24,6 & 20,3 & 18,0 \\
\cline { 2 - 12 } & Media & 15,7 & 16,8 & 18,9 & 20,7 & 23,7 & 27,4 & 30,1 & 30,4 & 27,8 & 23,7 & 19,0 & 16,0 \\
\hline
\end{tabular}

Tabla 1: Promedio mensual y media de los días con tipo de tiempo Este y Oeste de Tmax para Lisboa, Madrid y Valencia. 


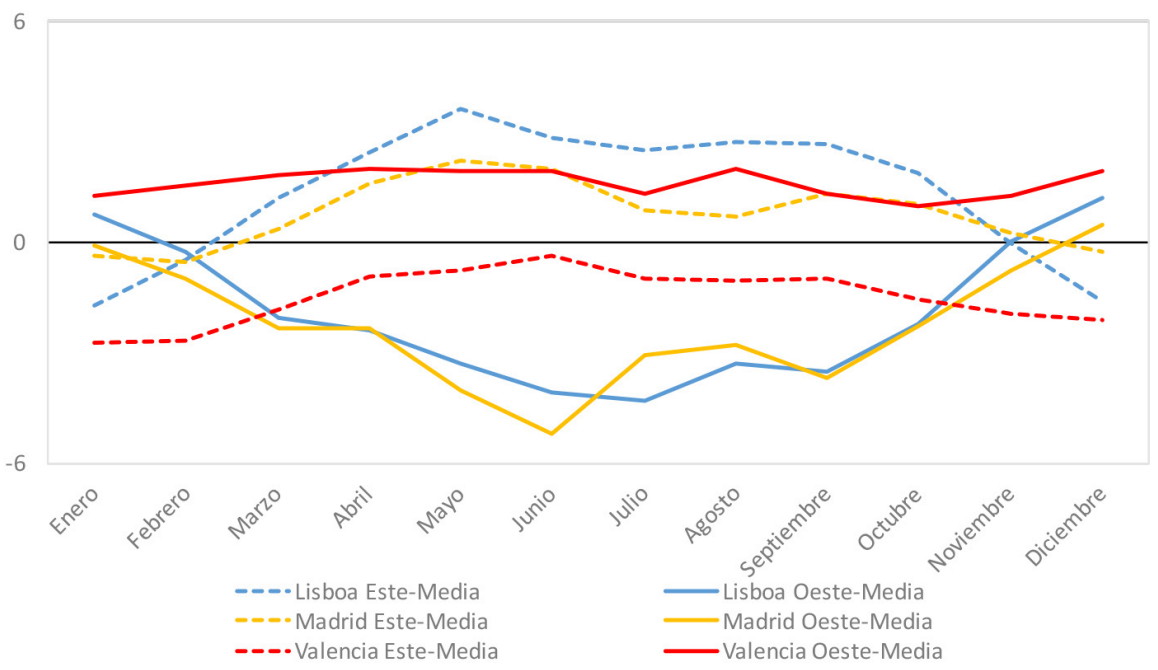

Fig 4: Anomalías mensuales de Tmax en condiciones de advecciones Este y Oeste en Madrid, Lisboa y Valencia
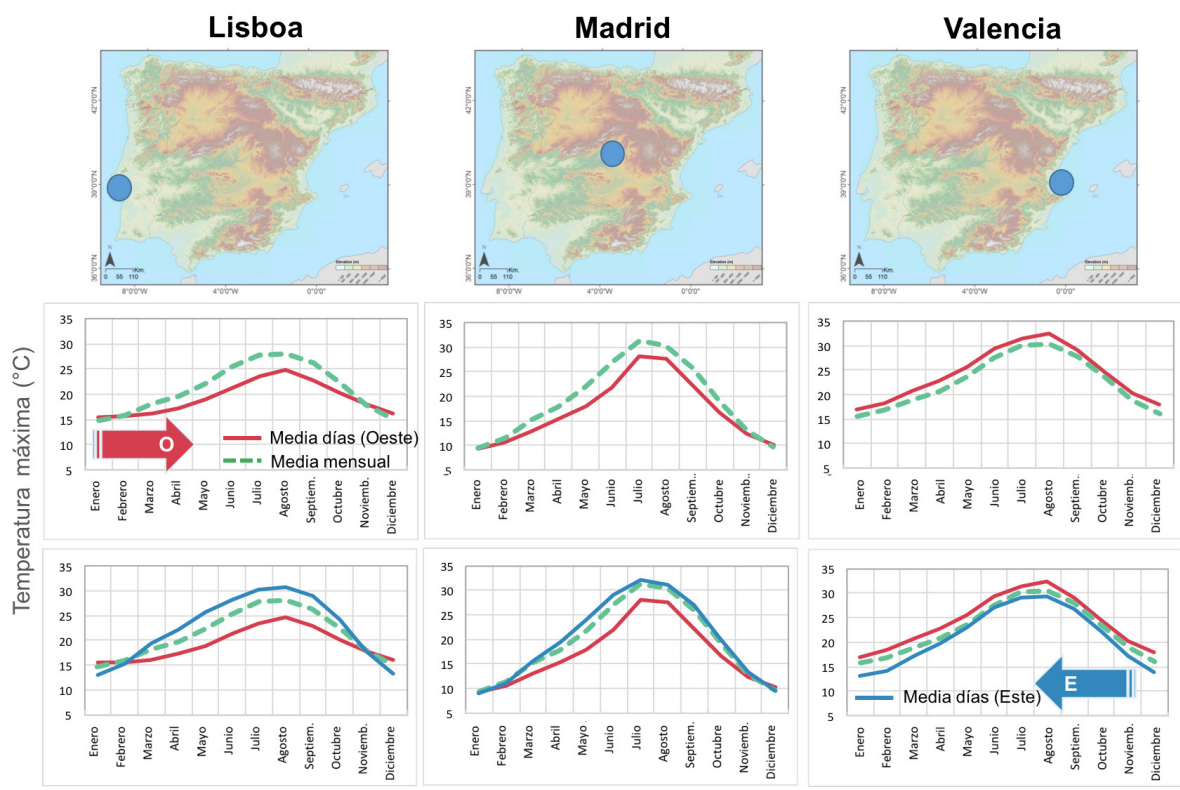

Fig 5: Promedio mensual (línea verde) y media de los días con tipo de tiempo Este (linea azul) y Oeste (linea roja) de Tmax para Lisboa, Madrid y Valencia

Cuando tiene lugar la advección del Oeste (Fig. 5) la temperatura diurna (Tmax) de Lisboa desciende por debajo de la media mensual. De igual forma con flujo del Oeste en Madrid hay un descenso de temperatura. Sin embargo, en Valencia tiene 
lugar un incremento de las temperaturas diurnas por encima de la media. Los días con flujo del Este se produce un descenso de las temperaturas por debajo del valor medio en Valencia, y una vez superada la barrera montañosa, en Madrid y Lisboa se produce un aumento de las temperaturas.

\section{CONCLUSIONES}

Se ha detectado un efecto foehn que afecta a la Península Ibérica en presencia de flujos zonales del Este y Oeste que atribuimos a la disposición del relieve, particularmente Sistema Ibérico y Sub-bético, paralelo a la costa mediterránea, y que implica que el comportamiento del interior peninsular se asemeje a la costa atlántica. En condiciones de advecciones Este la temperatura diurna desciende en la fachada mediterránea, y aumenta en el interior y costa atlántica; en condiciones de flujo del Oeste la temperatura diurna desciende en la costa atlántica y en el interior peninsular, y aumenta en la costa mediterránea.

El fenómeno afecta más a las temperaturas diurnas (Tmax) que nocturnas (Tmin), y varía entre meses, siendo más notable sus efectos fuera de los meses más fríos. Debido a que sus efectos son más evidentes en Tmax que en Tmin, se sugiere que la distribución de Tmax en la Península Ibérica se encuentra más influenciada que Tmin por la circulación general de la atmosfera, mientras las temperaturas nocturnas (Tmin) podrían depender en mayor grado de otros factores de escala local en las condiciones atmosféricas de advecciones zonales Este y Oeste analizadas.

\section{AGRADECIMIENTOS}

Ministerio de Ciencia e Innovación, Gobierno de España, Proyecto DESEMON, CGL2014-52135-C3-3-R. Gobierno Regional de Aragón DGA-FSE (Grupo de Investigación Consolidado “Clima, Agua, Cambio Global y Sistemas Naturales"). Dhais Peña-Angulo es becaria de doctorado del Programa FPI del Ministerio de Ciencia e Innovación. Datos climáticos originales cedidos por AEMet.

\section{REFERENCIAS}

Cortesi, N.; Trigo, R.M.; González-Hidalgo, J.C.; Ramos, A.M.(2013a). Modelling monthly precipitation with circulation weather types for a dense network os stations over Iberian. Hydrology and Earth System Science, 17, 665-678.

Cortesi, N.; González-Hidalgo, J.C.; Trigo, R.M.; Ramos, A.M. (2013b). Weather types and spatial variability of precipitation in the Iberian Peninsula. International Journal of Climatology, 34, 2661-2677.

Fernández-Montes, S.; Seubert, S.; Rodrigo, F.S.; Hertig, E. (2012). Wintertime circulation types over the Iberian Peninsula: long-term variability and relationships with weather extremes. Climate Research, 53, 205-227.

Gaffin, D.M. (2002). Unexpected warming induced by foehn winds in the lee of the Smoky Mountains. Weather and Forecasting, 17, 907-915.

Gonzalez-Hidalgo, J. C.; Peña-Angulo, D.; Brunetti, M.; Cortesi, N. (2015). MOTEDAS: a new monthly temperature database for mainland Spain and the 
trend in temperature (1951-2010). International Journal of Climatology, 35, 4444-4463.

Hojlund Pedersen, S.; Liston, G.E.; Tamstorf, M.P.; Westergaard-Nielsen, A.; Martin Schmidt, N. (2015). Quantifying Episodic Snowmelt Events in Arctic Ecosystems. Ecosystems, 18, 839-856 .

Jenkinson, A.F.; Collison, F.P. (1977). An initial climatology of Gales over the North Sea. Synoptic Climatology Branch Memorandum, 62, 18 Pages. Meteorological Office, Bracknell.

Jin, Y.; Randerson, J.T.; Faivre, N.; Capps, S.; Hall, A.; Goulden, M. (2014). Contrasting controls on wildland fires in Southern California during periods with and without Santa Ana winds. Journal of Geophysical Research-Biogeosciences, $119,432-450$.

Klistler, R.; Kalnay, E.; Collins, W.; Saha, S.; White, G.; Woollen, J.; Chelliah, M.; Ebisuzaki, W.; Kanamitsu, M.; Kousky, V.; Van Den Dool, H.; Jenne, R.; Fiorino, M. (2001). The NCEP-NCAR 50-year reanalysis: monthly means CD-ROM and documentation. Bull. Am. Meteorol. Soc. 82, 247-267.

Li, X.; Xia, X.; Wang, L.; Cai, R.; Zhao, L.; Feng, Z.; Feng, Z.; Ren, Q.; Zhao, K. (2015). The role of foehn in the formation of heavy air pollution events in Urumqi, China. Journal of Geophysical Research-Atmospheres, 120, 5371-5384.

Martín-Vide, J. (2002). Aplicación de la clasificación sinóptica de Jeckinson and Collison. En: Cuadrat, J.M.; Vicente, S.M.y Saz, M.A. (Eds.). La información climática como herramienta de gestión ambiental, pp. 123-127. Universidad de Zaragoza.

Natale, P.; Anfossi, D.; Cassardo, C. (1999). Analysis of an anomalous case of high air pollution concentration in Turin after a foehn event. International Journal of Enviroment and Pollution, 11, 147-164.

Peña-Angulo, D.; Trigo, R.; Cortesi, N.; González-Hidalgo, J.C. (2016). The influence of weather types on the monthly average máximum and mínimum temperatures in the Iberian Peninsula. Atmospheric Research, 178-179, 217-230.

Santurtún, A.; González-Hidalgo, J.C.; Sánchez-Lorenzo, A.; Zarrabeitia, M.T. (2015). Surface ozone concentration trends and its relationship with weather types in Spain (2001-2010). Atmospheric Environment, 101, 10-22.

Seluchi, M. E.; Norte, F. A.; Satyamurty, P. Chan Chou, S. (2003). Analysis of Three Situations of the Foehn Effect over the Andes (Zonda Wind) Using the EtaCPTEC Regional Model. Weather and Forecasting, 18, 481-501.

Sharples, J.J.; Mills, G.A.; McRae, R.; (2010). Foehn-Like Winds and Elevated Fire Danger Conditions in Southeastern Australia. Journal of Aplied Meteorology and Climatology, 49: 1067-1095.

Soderberg, S. and Parmhed, O. (2006). Numerical modelling of katabatic flow over a melting outflow glacier. Boundary-Layer Meteorology, 120, 509-534.

Spellman, G. (2000). The application of and objetive weather-typing system to the Iberian península. Weather, 55, 375-385. 
Takane, Y.; Kusaka, H. (2011). Formation Mechanisms of the Extreme High Surface Air Temperature of 40.9 degrees C observed in the Tokyo metropolitan area: Considerations of dynamic foehn and foehn-like wind. Journal applied meteorology and climatology, 50, 1827-1841.

Trigo, R.M.; DaCamara, C.C. (2000). Circulation weather types and their influence on the precipitation regime in Portugal. International Journal of Climatology, 20, 1559-1581. 